Ana Gabriela Macas-Macas; Darwin Gabriel García-Herrera; María Isabel Álvarez-Lozano Juan Carlos Erazo-Álvarez

http://dx.doi.org/10.35381/r.k.v5i5.1044

\title{
Dino Tim y el desarrollo lógico matemático en niños de Educación Inicial
}

\section{DinoTim and logical mathematical development in Early Childhood Education children}

\author{
Ana Gabriela Macas-Macas \\ ana.macas.22@est.ucacue.edu.ec \\ Universidad Católica de Cuenca, Machala \\ Ecuador \\ https://orcid.org/0000-0001-5790-712X \\ Darwin Gabriel García-Herrera \\ dggarciah@ucacue.edu.ec \\ Universidad Católica de Cuenca, Azogues \\ Ecuador \\ https://orcid.org/0000-0001-6813-8100 \\ María Isabel Álvarez-Lozano \\ mialvarezl@ucacue.edu.ec \\ Universidad Católica de Cuenca, Biblián \\ Ecuador \\ https://orcid.org/0000-0001-8029-1933 \\ Juan Carlos Erazo-Álvarez \\ jcerazo@ucacue.edu.ec \\ Universidad Católica de Cuenca, Cuenca \\ Ecuador \\ https://orcid.org/0000-0001-6480-2270
}

Recibido: 15 de septiembre de 2020

Revisado: 19 de octubre de 2020

Aprobado: 15 de noviembre de 2020

Publicado: 01 de diciembre de 2020 
Revista Arbitrada Interdisciplinaria KOINONIA

Año 2020. Vol V. N5. Especial II: Educación

Hecho el depósito de Ley: FA2016000010

ISSN: 2542-3088

FUNDACIÓN KOINONIA (F.K). Santa Ana de Coro. Venezuela.

Ana Gabriela Macas-Macas; Darwin Gabriel García-Herrera; María Isabel Álvarez-Lozano Juan Carlos Erazo-Álvarez

\title{
RESUMEN
}

El objetivo fue analizar el Dino Tim y el desarrollo lógico matemático en niños de Educación Inicial de un centro de educación del Ecuador. Se desarrolló desde una metodología mixta, combinándose resultados cuantitativos y cualitativos de alcance descriptivo con diseño no experimental, generándose un proceso observatorio exploratorio a través de encuesta y entrevista semi estructurada. La muestra poblacional estuvo conformada por 8 padres y 6 docentes de la escuela CEPAD "Sueños de David", ubicada en la parroquia Tarqui, al norte de Guayaquil, provincia de Guayas, Ecuador. En base a las encuestas se determina que los niños emplean herramientas de tecnología avanzada para el fortalecimiento y desarrollo de destrezas en lógica matemática una de las materias más temidas por los estudiantes, sin embargo, tanto maestros como estudiantes no conocían Dino Tim. Los docentes no están actualizados con la nueva tecnología por cuanto se les dificulta realizar ingresos o descargar programas.

Descriptores: Matemático; tecnología educacional; programa informático didáctico. (Palabras tomadas del Tesauro UNESCO).

\begin{abstract}
The objective was to analyze the Dino Tim and the mathematical logic development in Early Childhood Education children from an education center in Ecuador. It was developed from a mixed methodology, combining quantitative and qualitative results of descriptive scope with a non-experimental design, generating an observatory-exploratory process through a survey and semi-structured interview. The population sample consisted of 8 parents and 6 teachers from the CEPAD "Sueños de David" school, located in the Tarqui parish, north of Guayaquil, Guayas province, Ecuador. Based on the surveys, it is determined that children use advanced technology tools to strengthen and develop skills in mathematical logic, one of the subjects most feared by students, however, both teachers and students did not know Dino Tim. Teachers are not up to date with new technology because it is difficult for them to make income or download programs.
\end{abstract}

Descriptors: Mathematicians; educational technology; educational software. (Words taken from the UNESCO Thesaurus). 


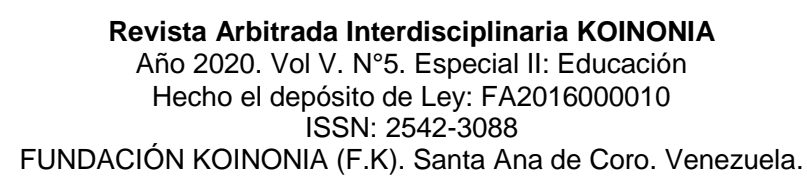

Ana Gabriela Macas-Macas; Darwin Gabriel García-Herrera; María Isabel Álvarez-Lozano Juan Carlos Erazo-Álvarez

\section{INTRODUCCIÓN}

La tecnología ha avanzado rápidamente, desde sus comienzos ha evolucionado el internet y para que este sea implementado el CERN decidió permitir que el software se encuentre a dominio público, fue patrimonio de todas las empresas y gobiernos en donde invirtieron grandes sumas de dinero para que este medio de comunicación sea posible. La Web es un espacio virtual en el cuál interactúan padres de familia, profesores, estudiantes, investigadores entre otros, ellos permiten conocer su información personal, laboral y contenidos en redes sociales, dan sus aportes para el público en general y a la vez consumen contenidos científicos, educativos, técnicos y teóricos que están online y son de fácil acceso.

El medio tecnológico se ha incrementado en los entornos personales, laborales, sociales y educativos. Las escuelas son muy importantes para los jóvenes del mañana, ya que la tecnología avanza día a día y requiere conocimientos desde la etapa preescolar, es lamentable ver a docentes con escasa alfabetización digital, falta de cultura e inadecuada formación docente que evita el correcto manejo de las TIC en la institución educativa. Con el paso del tiempo la tecnología se ha incluido en el medio educativo, por tal razón existe necesidades, como implementar las herramientas tecnológicas en las instituciones educativas, el cual requiere que los docentes se adapten a nuevas estrategias metodológicas para el beneficio mismo y del estudiante.

El objetivo fue analizar el Dino Tim y el desarrollo lógico matemático en niños de Educación Inicial de un centro de educación del Ecuador.

\section{Referencial teórico}

El Ministerio de Educación de Ecuador modificó el Currículo de Educación Inicial con el fin de Cambiar el proceso de enseñanza y aprendizaje de los niños de 3 a 5 años. En la actualidad muchos niños que asisten a Educación Inicial presentan dificultad en la comprensión de conocimientos lógicos matemáticos, esto se debe a la falta de estímulo al momento de realizar actividades, aplicación errónea en la metodología de trabajo lo 


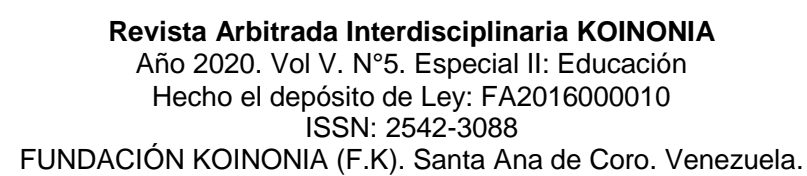

Ana Gabriela Macas-Macas; Darwin Gabriel García-Herrera; María Isabel Álvarez-Lozano Juan Carlos Erazo-Álvarez

que hace que el estudiante pierda interés por explorar, crear, innovar y comprender lo que hay a su alrededor, no se relacionan con otras personas lo que impide el desarrollo del pensamiento cognitivo o las experiencias de aprendizaje no son desafiantes.

Al realizar el proyecto investigativo de la app como medio de fortalecimiento en el aprendizaje en niños de inicial, tomé en cuenta variedad de investigaciones que hacen hincapié al tema de las Tics en preescolar, a nivel nacional e internacional, como es el caso (De-La-Serna-Tuya, et al., 2018), en donde afirman que las TIC son necesarias tanto en forma directa e indirecta, exige a los docentes a tener cambios en la práctica y a la vez realiza reestructuración educativa en todos los ciclos, al igual que las tecnologías debidamente instaladas en las aulas de clase. En México niños de 3 a 5 años están obligados asistir a las aulas de clase como lo establece la legislación educativa y que el uso de las tecnologías, innovaciones pedagógicas, gestión y diseño escolar permiten avanzar a mayor rapidez que sus profesores en el aprendizaje de apps digitales, incurriendo en la calidad del sistema educativo.

En el año 2011 American Academy of Pediatrics relata que aproximadamente el $90 \%$ de niños de edad temprana están relacionados con algún tipo de tecnología, pero existe mucha libertad de parte de los padres de familia al dejar que sus hijos trabajen con aplicaciones sin la debida precaución. (Guel-Silva, 2015), explica que las TIC ayudan al desarrollo de la competitividad preescolar, conforme se ha observado el crecimiento y desarrollo cognitivo de los niños al manejar aparatos o aplicaciones tecnológicas.

Otras investigaciones tomadas a consideración para el uso de la Tecnología de Información y comunicación hacia la integración curricular de Inicial es la de (AvendañoPorras, 2015), en la cual menciona que la tecnología permite que la educación pueda ser accesible para toda la población, dejando que exista desarrollo cognitivo en los estudiantes para mejorar sus competencias científicas y a su vez mejorar el desempeño académico en el área matemática.

Por otro lado, (Arellano, 2019) expresa que en Tabasco la Escuela David P. Ausubel, tiene 3 niveles: preescolar, primaria y secundaria, En el nivel de primaria muchos jóvenes 


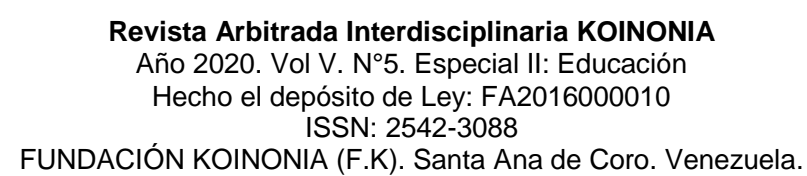

Ana Gabriela Macas-Macas; Darwin Gabriel García-Herrera; María Isabel Álvarez-Lozano Juan Carlos Erazo-Álvarez

saben usar correctamente las tecnologías como son: ordenadores, tabletas, laptops, dispositivos Android, esto no es nada complejo para ellos por lo que tienen acceso a celulares y con mucha frecuencia lo utilizan para jugar online, debido a esto en preescolar aplicaron los medios tecnológicos para desarrollar competencias en matemáticas debido al bajo rendimiento que tenían en comparación a otras escuelas del municipio y notaron que brinda mayor facilidad a los niños preescolares. Por ello es importante el uso de las Tics en la sociedad, ya que esta era exige políticas tecnológicas, lo cual resulta positivo tanto para el docente como el infante, además es necesario enfocarse a la actualidad que estamos viviendo, todo lo que concierne a educación se está enseñando vía online debido a la pandemia.

Así mismo, (Hamón \& Portela, 2017) dan a conocer que las apps educativas son parte de las Tics, conjuntos de técnicas y dispositivos avanzados de transmisión digitalizada en la información. Se refieren como tecnología a laptop, televisor y celulares, los cuales son de fácil acceso para los padres de familia, docentes y estudiantes.

En complemento (Comín-Hernando, 2015), afirma que las Tecnologías de la Información y de la Comunicación son necesarias para gestionar y transformar información, incluso al usar laptops o computadores que trabajan con softwares que permiten almacenar, crear, administrar, proteger, eliminar, borrar y modificar archivos. Lo nuevo de la tecnología facilita el acceso a aplicaciones didácticas y educativas en celulares, tabletas u ordenadores para niños que están en etapa preescolar hasta jóvenes universitarios.

Las grietas digitales son factores negativos para los niños de preescolar debido a la falta de aparatos tecnológicos, esto se debe a varios factores, tales como: situación económica, nivel académico, situación laboral, ubicación geográfica y la edad del padre de familia. En las escuelas públicas difícil encontrar tecnología avanzada, la cual las autoridades permitan usar para la práctica de niños de 4 a 5 años.

(Torres-Toukomidis \& Romero-Rodriguez, 2018) Aportan que se debe superar las limitaciones del aprendizaje de la vieja escuela que se basa en la memorización de contenidos para abrirse a nuevos espacios en los que desarrolle habilidades y 


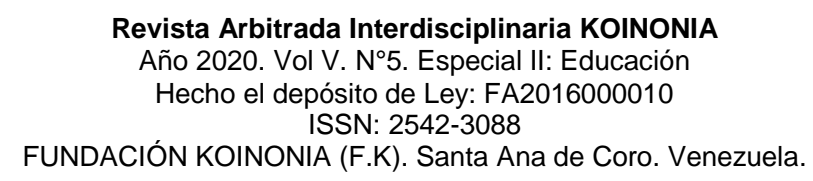

Ana Gabriela Macas-Macas; Darwin Gabriel García-Herrera; María Isabel Álvarez-Lozano Juan Carlos Erazo-Álvarez

competencias entrelazadas a aprender a aprender y a la apropiación de los conocimientos en profesores y niños a partir de las tecnologías y softwares multimedia. (De-Caso, et al., 2012), confirman que el aprendizaje de educación Inicial es de suma importancia ya que es la primera etapa la cuál es la base para la adquisición del conocimiento a futuro. Como docente tenemos la obligación de cuidar la enseñanza que impartimos a los niños de preescolar, ya que las competencias que se adquieran servirán para el resto de sus vidas en la inteligencia lógica matemática. Los educandos deben estar con actitud positiva para captar el mayor conocimiento posible, y al mismo tiempo evitar que se genere ansiedad o miedo al trabajar con números.

Diversas investigaciones han mostrado que la educación a nivel mundial se ha ido transformando y ha permitido la incorporación de la Tecnología de Información y Comunicación por medio de las Instituciones virtuales o apps dinámicas, ya que es esencial para la sociedad. Algunas Apps se las usan como complemento del educador dentro de las aulas de clase, como apoyo escolar, como reemplazo de los maestros particulares, otros como medios o herramientas que facilitan la realización de materias específicas o ejercicios matemáticos. Para determinar que aplicaciones son descargadas frecuentemente se utilizó una de las líderes del monitoreo y análisis de mercado como es el ranking de descargas de la consultora App Annie, en la cual se observó que el mayor índice de descargas son aplicaciones educativas de la tienda Android en Google Play (Franceschin, 2017).

La nueva metodología de impartir clases por medio de plataformas online ha hecho que muchos padres de familia y docentes realicen críticas poco constructivas, ya que dudan de su eficiencia y eficacia en el aprendizaje del alumno, a su vez (Cano, 2012), expresa que la calidad académica disminuye, lo que produce cambios en los paradigmas didácticos $\mathrm{y}$ formativos de docentes y educandos. El sistema educativo del siglo XXI enfrenta grandes desafíos con el uso de las TIC, las aspiraciones del ministerio de educación son proporcionar los conocimientos a jóvenes y los más pequeños de 


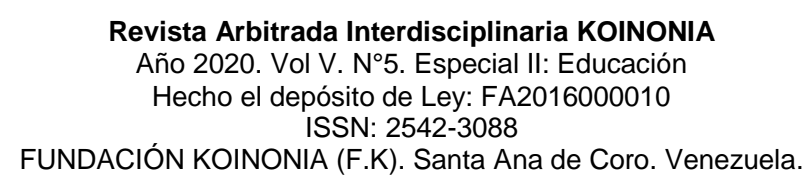

Ana Gabriela Macas-Macas; Darwin Gabriel García-Herrera; María Isabel Álvarez-Lozano Juan Carlos Erazo-Álvarez

educación inicial, con la finalidad de que les permita tener éxitos y a la vez tengan competitividad personal y académica.

El efecto que surge a través del uso de las TIC es cambiar la enseñanza de la escuela tradicional y convertirlos en modelos innovadores de aprendizaje enfocado en el alumno por medio de la adquisición de conocimientos virtual. Se debe ampliar los recursos, las estrategias didácticas para mejorar el aprendizaje del niño, hacer uso de la tecnología con el fin de obtener conocimientos que serán ventajoso al momento de ingresar a la escuela primaria.

Para ello el educador debe estudiar la app con el fin de verificar si tiene recursos importantes para la adquisición del conocimiento infantil, y luego inducir de manera eficiente. En esta época se requiere que los niños generalicen los entornos mediáticos, especialmente la tecnología multimedia, como son los videos educativos, aplicaciones educativas, plataformas tecnológicas, entre otros. Los niños de Inicial se pueden beneficiar del uso de computadoras, tabletas o celulares únicamente si se realiza de la forma correcta.

El ministerio de Educación es el encargado de la educación nacional, tiene el compromiso de brindar a los niños una educación de calidad, igualdad y oportunidades, para ello elaboraron un Currículo de Educación Inicial la cual surge para tener en cuenta la diversidad social, cultural y personal. El (Ministerio de educación del Ecuador [MINEDUC], 2014), se ha plasmado la importancia del lugar en el que habita el infante desde sus inicios de vida, ya que son factores que inciden en su desarrollo para el diario vivir, por ello se considera importante el ambiente y recursos proporcionados para el aprendizaje. Vygotsky considera que el desarrollo cognitivo es inseparable de la sociedad, plantea que los aprendizajes y el desarrollo psicológico infantil no van juntos, sino que existe un desfase normal y permite que surja el aprendizaje que se da desde el nacimiento.

El sistema educativo en Ecuador avanza con mayor rapidez en los medios tecnológicos, para la educación temprana en matemáticas es indispensable usar herramientas de fácil 


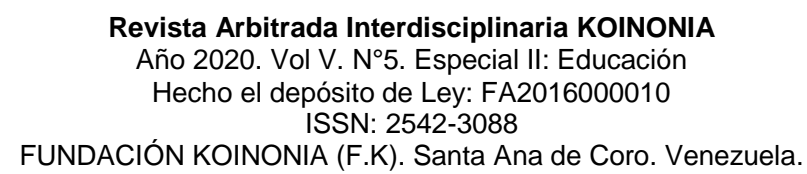

Ana Gabriela Macas-Macas; Darwin Gabriel García-Herrera; María Isabel Álvarez-Lozano Juan Carlos Erazo-Álvarez

acceso, la cual facilite la adquisición de conocimientos por medio de la interactividad y animación de las apps. En el presente trabajo se utiliza como base el currículo de educación inicial de (MINEDUC, 2014), define lo que se quiere conseguir, los objetivos y metas educativas, además de los medios y herramientas para la evaluación preescolar. La educación inicial es un proceso que requiere compañía para que haya buen desarrollo integral en los niños menores de 5 años, que busca potenciar el aprendizaje y promover el bienestar común, sin olvidar la respectiva responsabilidad que tiene la familia y sociedad. Por ello se realizará la aplicación de un test y pre-test, los que permitirán descubrir el tipo de dificultad que presenta el estudiante en el área de matemática y a la vez ayudar a niños de 4 a 5 años a mejorar el razonamiento lógico-matemático combinando la dinámica, imaginación e interés del niño por aprender.

Para el buen aprendizaje del niño intervienen tres individuos, padre de familia, docente y estudiante. Es fundamental y de mucha importancia desempeñar bien el rol de padre ya que el niño desde casa va con conocimientos básicos como son los valores, principios comportamientos genuinos y dimensiones sociales, por este motivo el representante debe acompañar en la enseñanza y dedicarle el tiempo necesario para que los niños generen confianza y aceptación, el docente se encarga de proporcionar el debido conocimiento y a la vez ser guía y cómplice en el transcurso del ciclo Inicial usando aptitudes que le ayudará a comprender de manera más sencilla las diversas materias, y el estudiante es quien va absorber el conocimiento impartido por el profesor.

\section{METODOLOGÍA}

La investigación se desarrolló desde una metodología mixta, combinándose resultados cuantitativos y cualitativos de alcance descriptivo con diseño no experimental, generándose un proceso observatorio - exploratorio a través de encuesta y entrevista semi estructurada. La muestra poblacional estuvo conformada por 8 padres y 6 docentes de la escuela CEPAD "Sueños de David", ubicada en la parroquia Tarqui, al norte de 
Ana Gabriela Macas-Macas; Darwin Gabriel García-Herrera; María Isabel Álvarez-Lozano Juan Carlos Erazo-Álvarez

Guayaquil, provincia de Guayas, Ecuador. La recolección de información se realizó del siguiente modo:

Para los datos cuantitativos, se aplicó como técnica la encuesta y cuestionario en escala Likert de 14 ítems, el cual fue validado por juicio de expertos y confiabilidad de Alfa de Cronbach con resultado de 0,89 siendo confiable para su aplicación.

Para la información cualitativa, se empleó como técnica de recolección de información, la entrevista, mediante cuestionario semi estructurado con preguntas abiertas, diseñadas a partir del proceso de observación y análisis de los investigadores sobre la realidad de estudio, así se generó un conversatorio que permitió generar categorías emergentes sobre el tema abordado.

Los datos cuantitativos recopilados fueron procesados en función de la estadística descriptiva y chi cuadrado de Pearson, mientras que los cualitativos, se empleó, el análisis de contenido.

\section{RESULTADOS}

A continuación, se detallas los principales resultados:

Tabla 1.

Eficacia en los ambientes de aprendizaje.

\begin{tabular}{cccccc}
\hline & Frecuencia & Porcentaje & $\begin{array}{c}\text { Porcentaje } \\
\text { válido }\end{array}$ & $\begin{array}{c}\text { Porcentaje } \\
\text { acumulado }\end{array}$ \\
\hline \multirow{3}{*}{ Válido } & Neutro & 1 & 16.7 & 16.7 & 16.7 \\
& De acuerdo & 3 & 50.0 & 50.0 & 66.7 \\
& Totalmente de acuerdo & 2 & 33.3 & 33.3 & 100.0 \\
& Total & 6 & 100.0 & 100.0 & \\
\hline
\end{tabular}

Fuente: Elaboración propia. 
Ana Gabriela Macas-Macas; Darwin Gabriel García-Herrera; María Isabel Álvarez-Lozano Juan Carlos Erazo-Álvarez

Para el análisis de datos se usó información recopilada de la Tabla 1 en donde visualizamos que aproximadamente el $83.3 \%$ de los docentes están de acuerdo y totalmente de acuerdo con el criterio de que son eficaces los ambientes de aprendizaje para la adquisición de conocimientos en el niño de Inicial.

Tabla 2.

Metodologías de Gamificación.

\begin{tabular}{cccccc}
\hline & & Frecuencia & Porcentaje & $\begin{array}{c}\text { Porcentaje } \\
\text { válido }\end{array}$ & $\begin{array}{c}\text { Porcentaje } \\
\text { acumulado }\end{array}$ \\
\hline \multirow{3}{*}{ Válido } & Neutro & 2 & 33.3 & 33.3 & 33.3 \\
& Acuerdo & 3 & 50.0 & 50.0 & 83.3 \\
& Totalmente de acuerdo & 1 & 16.7 & 16.7 & 100.0 \\
\hline
\end{tabular}

Fuente: Elaboración propia

En la tabla 2 los docentes demuestran que la Gamificación es muy útil para el proceso de aprendizaje. Se aprecia que el $50 \%$ de los docentes están de acuerdo con la metodología de Gamificación, ya que mejora el proceso de enseñanza- aprendizaje lo que da como resultado positivo en el uso de juegos didácticos que es lo que se está aplicando en esta investigación. 
Revista Arbitrada Interdisciplinaria KOINONIA

Año 2020. Vol V. N5. Especial II: Educación

Hecho el depósito de Ley: FA2016000010

ISSN: 2542-3088

FUNDACIÓN KOINONIA (F.K). Santa Ana de Coro. Venezuela.

Ana Gabriela Macas-Macas; Darwin Gabriel García-Herrera; María Isabel Álvarez-Lozano Juan Carlos Erazo-Álvarez

\section{Tabla 3.}

Relación de tecnologías y Gamificación.

\begin{tabular}{|c|c|c|c|c|c|}
\hline & & \multicolumn{3}{|c|}{ Metodología de Gamificación } & \multirow[b]{2}{*}{ Total } \\
\hline & & Neutro & Acuerdo & $\begin{array}{l}\text { Totalmente } \\
\text { de acuerdo }\end{array}$ & \\
\hline \multirow{6}{*}{$\begin{array}{c}\text { Ambientes de } \\
\text { aprendizaje } \\
\text { tecnológicos }\end{array}$} & \multirow{2}{*}{ Neutro } & 1 & 0 & 0 & 1 \\
\hline & & .3 & .5 & .2 & 1.0 \\
\hline & \multirow{2}{*}{$\begin{array}{c}\text { De } \\
\text { acuerdo }\end{array}$} & 1 & 1 & 1 & 3 \\
\hline & & 1.0 & 1.5 & .5 & 3.0 \\
\hline & \multirow{2}{*}{$\begin{array}{l}\text { Totalmente } \\
\text { de acuerdo }\end{array}$} & 0 & 2 & 0 & 2 \\
\hline & & .7 & 1.0 & .3 & 2.0 \\
\hline \multirow{2}{*}{\multicolumn{2}{|c|}{ Total }} & 2 & 3 & 1 & 6 \\
\hline & & 2.0 & 3.0 & 1.0 & 6.0 \\
\hline
\end{tabular}

Pruebas de chi-cuadrado

\begin{tabular}{cccc}
\hline & Valor & df & Significación asintótica (bilateral) \\
Chi-cuadrado de Pearson & $4.667^{\mathrm{a}}$ & 4 & .323 \\
Razón de verosimilitud & 5.545 & 4 & .236 \\
Asociación lineal por lineal & .848 & 1 & .357 \\
N de casos válidos & 6 & & \\
\hline
\end{tabular}

a. 9 casillas (100.0\%) han esperado un recuento menor que 5. El recuento mínimo esperado es .17.

Fuente: Elaboración propia.

De acuerdo con el criterio del autor el valor del chi cuadrado hay que mirarlo con cautela, se debe observar en la tabla 5 el grado de significación que es 0,323 $>0,05$, se asume la hipótesis nula, atendiendo que 9 de las casillas tienen frecuencias esperadas menores a 5. Para las siguientes tablas y gráficos es de suma importancia saber el criterio de los padres de familia sobre los métodos de enseñanza y recursos que usan los docentes con sus hijos en la institución. 
Revista Arbitrada Interdisciplinaria KOINONIA

Año 2020. Vol V. N5. Especial II: Educación

Hecho el depósito de Ley: FA2016000010

ISSN: 2542-3088

FUNDACIÓN KOINONIA (F.K). Santa Ana de Coro. Venezuela.

Ana Gabriela Macas-Macas; Darwin Gabriel García-Herrera; María Isabel Álvarez-Lozano Juan Carlos Erazo-Álvarez

\section{Tabla 4.}

Destreza con apps didáctica.

\begin{tabular}{cccccc}
\hline & & Frecuencia & Porcentaje & $\begin{array}{c}\text { Porcentaje } \\
\text { válido }\end{array}$ & $\begin{array}{c}\text { Porcentaje } \\
\text { acumulado }\end{array}$ \\
\hline \multirow{3}{*}{ Válido } & Neutro & 1 & 12.5 & 12.5 & 12.5 \\
& Acuerdo & 4 & 50.0 & 50.0 & 62.5 \\
& Totalmente de acuerdo & 3 & 37.5 & 37.5 & 100.0 \\
& Total & 8 & 100.0 & 100.0 & \\
\hline
\end{tabular}

Fuente: Elaboración propia.

En la Tabla 4 podemos visualizar que los padres de familia piensan que las apps ayudan a desarrollar destrezas en el área de matemáticas. El 50\% de los padres indican estar totalmente de acuerdo con las aplicaciones didácticas por lo que expresan que los niños van a obtener más conocimientos.

\section{Tabla 5.}

Dino Tim para fortalecer el aprendizaje del niño.

\begin{tabular}{llrrrr}
\hline & Frecuencia & Porcentaje & $\begin{array}{c}\text { Porcentaje } \\
\text { válido }\end{array}$ & $\begin{array}{c}\text { Porcentaje } \\
\text { acumulado }\end{array}$ \\
\hline \multirow{3}{*}{ Válido } & Acuerdo & 4 & 50.0 & 50.0 & 50.0 \\
& Totalmente de acuerdo & 4 & 50.0 & 50.0 & 100.0 \\
& Total & 8 & 100.0 & 100.0 & \\
\hline
\end{tabular}

Fuente: Elaboración propia.

En la Tabla 5 los padres de familia expresan que están dispuestos a integrar la aplicación con el fin de ver mejoras en su hijo. Un $50 \%$ de los encuestados mencionan que están totalmente de acuerdo con Dino Tim para el mejoramiento de lógica matemáticas en sus hijos de nivel preescolar. 
Revista Arbitrada Interdisciplinaria KOINONIA

Año 2020. Vol V. N5. Especial II: Educación

Hecho el depósito de Ley: FA2016000010

ISSN: 2542-3088

FUNDACIÓN KOINONIA (F.K). Santa Ana de Coro. Venezuela.

Ana Gabriela Macas-Macas; Darwin Gabriel García-Herrera; María Isabel Álvarez-Lozano Juan Carlos Erazo-Álvarez

\section{Tabla 6.}

Análisis cualitativo Docentes.

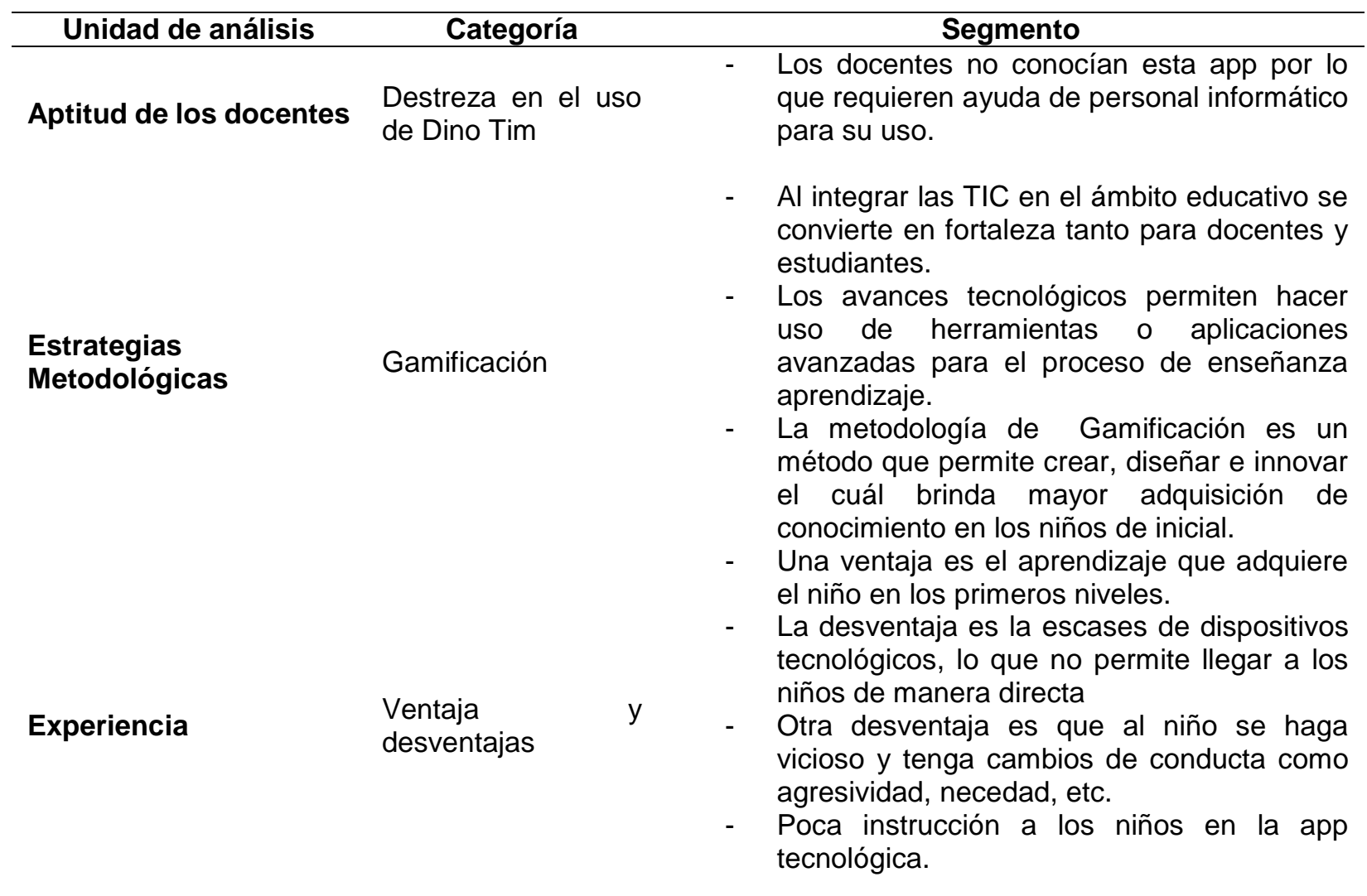

Fuente: Elaboración propia.

La Tabla 6 muestra las entrevistas realizadas a docentes en las que se pudo observar que 4 de los 6 entrevistados están de acuerdo que Dino Tim es una herramienta tecnológica innovadora que dándole el uso adecuado servirá para el fortalecimiento de lógica matemática en los niños de Inicial II. 
Revista Arbitrada Interdisciplinaria KOINONIA

Año 2020. Vol V. N5. Especial II: Educación

Hecho el depósito de Ley: FA2016000010

ISSN: 2542-3088

FUNDACIÓN KOINONIA (F.K). Santa Ana de Coro. Venezuela.

Ana Gabriela Macas-Macas; Darwin Gabriel García-Herrera; María Isabel Álvarez-Lozano Juan Carlos Erazo-Álvarez

Tabla 7.

Análisis cualitativo en Representantes.

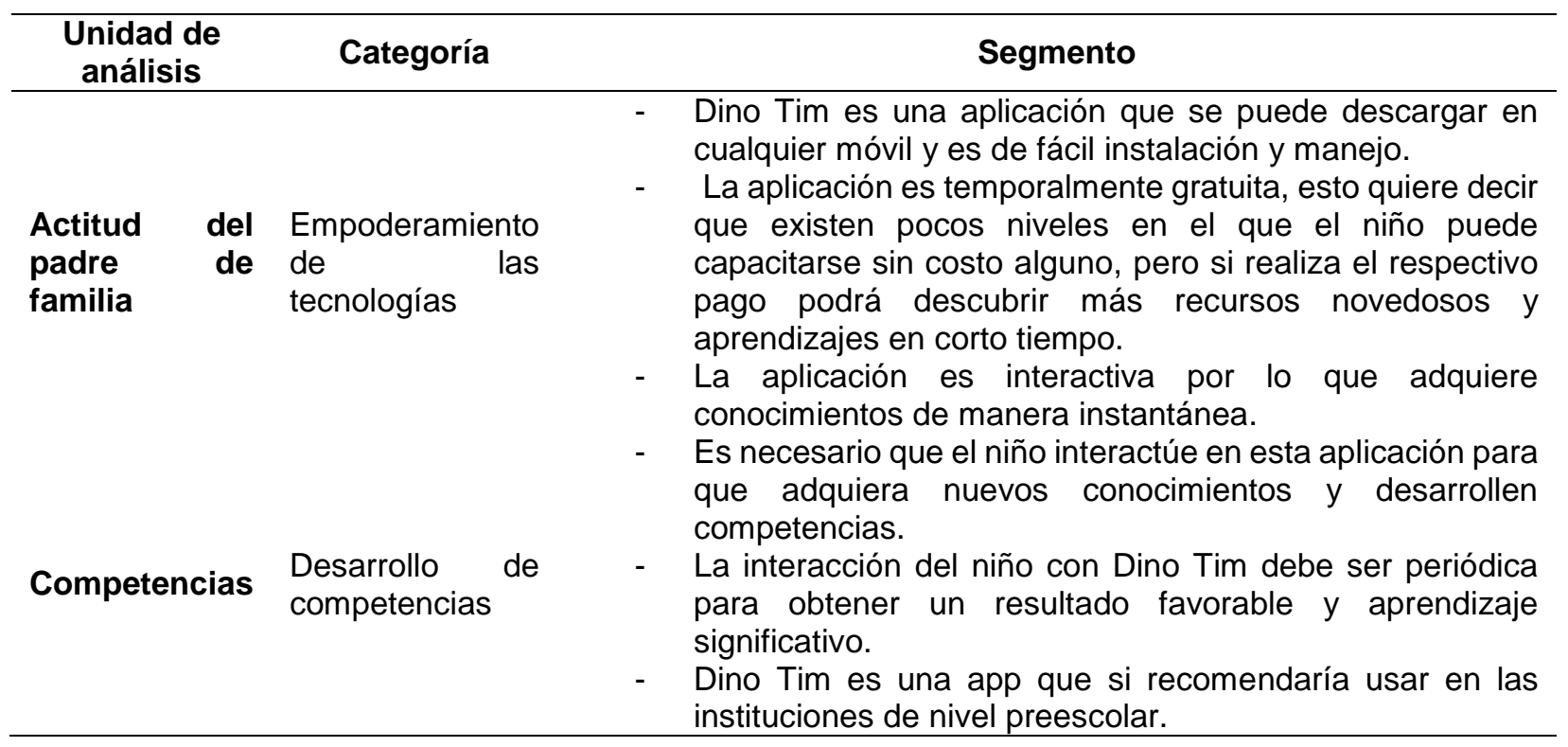

Fuente: Elaboración propia.

En base a las entrevistas realizadas se puede corroborar que 7 de los 8 padres de familia están de acuerdo que Dino Tim es una aplicación útil que permite fortalecer y desarrollar destrezas en lógica matemática en los niños de Inicial II, además la recomiendan por su fácil acceso por ende si cumple con los objetivos planteados.

\section{PROPUESTA}

Dentro del proceso de enseñanza-aprendizaje abarca diversos factores que son indispensables para el aprendizaje lógico del niño, en etapa preescolar son las bases para avanzar en los niveles posteriores. Por ello surge la necesidad de fortalecer el conocimiento lógico matemático en los niños de Inicial usando medios tecnológicos que permiten que el infante se interese más y a la vez se motive a través del uso de Dino Tim. Es de suma importancia la alfabetización en docentes y padres de familia, porque de ellos 
dependerá que tan capacitados estén los niños en los medios tecnológicos. La propuesta está dada por el siguiente esquema:

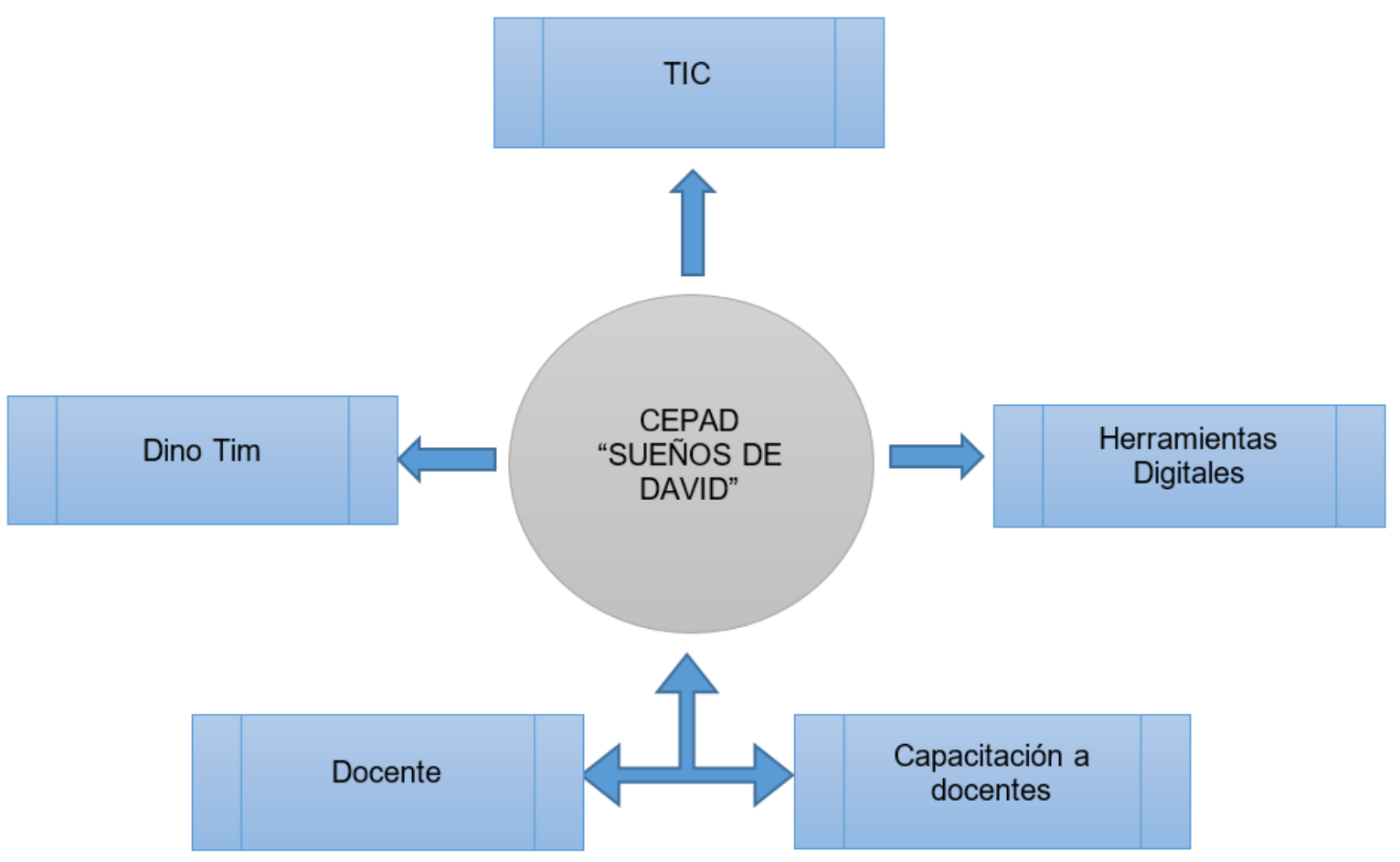

Figura 1. Propuesta uso Dino Tim en Educación Inicial.

Fuente: Elaboración propia.

1. Aplicación de las TIC como proceso de enseñanza.- Favorece a las escuelas de servicio público y privado en donde garantiza la preparación de las próximas generaciones por eso es importante aplicar las Tecnologías de Información y Comunicación, para ello se recomienda usar Dino Tim que es una app con recursos interactivos, dinámicos y atractivos que refuerzan la comprensión lógica 


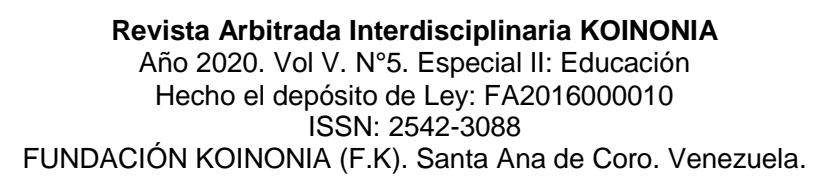

Ana Gabriela Macas-Macas; Darwin Gabriel García-Herrera; María Isabel Álvarez-Lozano Juan Carlos Erazo-Álvarez

matemática, además de permitir que el niño trabaje conjuntamente con sus compañeros y colabore con el profesor.

2. Contratar un docente informático que imparta la materia denominada Técnicas digitales. El profesional informático tiene arduos conocimientos en programación, robótica, software y hardware por ello es indispensable contar con un docente que imparta sus saberes a los docentes y niños de la unidad educativa CEPAD.

3. Capacitar a los docentes de Inicial para que exista menos alfabetización digital y puedan adquirir todos los conocimientos tecnológicos para la correcta aplicación y utilización de las Tic.

4. Implementar herramientas digitales: La rectora debería invertir parte de los recursos de la institución en adquisición de aparatos y softwares tecnológicos tales como: Computadoras, Tablet o Laptops, programas matemáticos, Dino Tim, Smartick entre otros, para que los niños tengan una educación productiva y de calidad, orientados a explorar, crear e imaginar conforme requiere la nueva era digital.

5. Incluir Dino Tim como guía de aprendizaje de lógica matemática para que el niño de etapa preescolar aprenda y mejore la rapidez, concentración y psicomotricidad con la app de reconocimiento de los números, colores y figuras geométricas de manera dinámica y divertida.

\section{CONCLUSIONES}

El niño debe descubrir y a la vez absorber todo el conocimiento posible de la aplicación con el fin de que su experiencia virtual sea eficaz para obtener éxito en el fortalecimiento de lógica matemática por medio de los recursos o herramientas tecnológicas a las que tiene acceso.

En base a las encuestas se determina que los niños emplean herramientas de tecnología avanzada para el fortalecimiento y desarrollo de destrezas en lógica matemática una de 
Ana Gabriela Macas-Macas; Darwin Gabriel García-Herrera; María Isabel Álvarez-Lozano Juan Carlos Erazo-Álvarez

las materias más temidas por los estudiantes, sin embargo, tanto maestros como estudiantes no conocían Dino Tim.

Los docentes no están actualizados con la nueva tecnología por cuanto se les dificulta realizar ingresos o descargar programas, por lo que se recomienda capacitaciones con un profesional informático que les enseñe detalladamente lo nuevo en aplicaciones o plataformas virtuales.

Los padres de familia están contentos al ver a sus pequeños aprender las matemáticas básicas, por cuanto este aprendizaje servirá para sus próximos niveles académicos.

\section{FINANCIAMIENTO}

No monetario

\section{AGRADECIMIENTO}

A la escuela CEPAD "Sueños de David", ubicada en la parroquia Tarqui, al norte de Guayaquil, provincia de Guayas, Ecuador; por apoyar el desarrollo de la investigación.

\section{REFERENCIAS CONSULTADAS}

Arellano, C. (2019). Desarrollo de Habilidades Matemáticas en alumnos de preescolar usando un ambiente de aprendizaje mezclado. [Development of Mathematical Skills in Preschool Student Using a Blended Learning Enviroment]. Juárez: Universidad Juarez Autónoma de Tabasco. Obtenido de http://ri.ujat.mx/handle/20.500.12107/3089

Avendaño-Porras, V. d. (2015). Implementación y uso de las tecnologías de información y la comunicación: en la Maseta Comitéca Tojolabal del estado de Chiapas. [Implementation and use of information and communication technologies: in the Maseta Comiteca Tojolabal of the state of Chiapas].. Chiapas: Centro Regional de Formación Docente e Investigación Educativa. Obtenido de https://n9.cl/0ujb5 
Revista Arbitrada Interdisciplinaria KOINONIA

Año 2020. Vol V. N5. Especial II: Educación

Hecho el depósito de Ley: FA2016000010

ISSN: 2542-3088

FUNDACIÓN KOINONIA (F.K). Santa Ana de Coro. Venezuela.

Ana Gabriela Macas-Macas; Darwin Gabriel García-Herrera; María Isabel Álvarez-Lozano Juan Carlos Erazo-Álvarez

Cano-Lassonde, O. (2012). Antecedentes internacionales y nacionales de las Tic a nivel superior: su trayectoria en panamá. [International and national antecedents of ICT at the higher level: its trajectory in panama]. Revista Electrónica "Actualidades Investigativas en Educación", 12(3),1-25.

Comín-Hernando, S. (2015). Apps e infancia. Estudio de las apps educativas para dispositivos móviles orientadas a la enseñanza infantil. [Apps and childhood. Study of educational applications for mobile devices aimed at children's education]. Master Thesis, Universidad Nacional de Educación a Distancia (España). Facultad de Educación. Obtenido de https://n9.cl//6dh

De-Caso, A. M., Blanco, J., \& Navas, G. (2012). Las TICS en segundo ciclo de educación infantil [ITC in the second cycle of early childhood education]. International Journal of Developmental and Educational Psychology, 1(1), 189-197.

De-La-Serna-Tuya, A. S., Gonzáles-Calleros, J. M., \& Navarro, Y. (2018). Diseño de App para el uso de la tablet en la enseñanza de preescolares. [App design for tablet use on preschool teaching]. Campos Virtuales, 7(1); 111-123.

Franceschin, T. (2017). ¿Qué aplicaciones móviles educativas son las más populares en América Latina? [What educational mobile applications are the most popular in Latin America?]. Obtenido de Edu4: https://n9.cl/rsip

Guel-Silva, G. (2015). Propuesta de aplicación educativa, para el proceso de enseñanza-aprendizaje en preescolares. [Educational application proposal for the teachinglearning process in preschool]. Puebla: Universidad Iberoamericana Puebla. Obtenido de https://n9.cl/czrmv

Hamón, E., \& Portela, A. (2017). Apps educativas como herramienta pedagógica para niños y niñas de grado segundo en el Colegio Sorrento LE.D. [Educational apps as a pedagogical tools for second grade boys and girls at Collage Sorrento LE.D]. Bogotá: Universidad de Libertadores. Obtenido de https://n9.cl/mt05d

Ministerio de educación del Ecuador [MINEDUC]. (2014). Curriculo educación inicial 2014. [Curriculum initial education 2014]. Obtenido de https://n9.cl/2itc6 
Revista Arbitrada Interdisciplinaria KOINONIA

Año 2020. Vol V. N5. Especial II: Educación

Hecho el depósito de Ley: FA2016000010

ISSN: 2542-3088

FUNDACIÓN KOINONIA (F.K). Santa Ana de Coro. Venezuela.

Ana Gabriela Macas-Macas; Darwin Gabriel García-Herrera; María Isabel Álvarez-Lozano Juan Carlos Erazo-Álvarez

Torres-Toukomidis, A., \& Romero-Rodriguez, L. (2018). Gamificaciòn en Iberoamérica. [Gamification in Latin America]. Quito: Universidad Abya-Yala. Obtenido de https://n9.cl/rijz9

(C2020 por los autores. Este artículo es de acceso abierto y distribuido según los términos y condiciones de la licencia Creative Commons Atribución-NoComercial-Compartirlgual 4.0 Internacional (CC BY-NC-SA 4.0) (https://creativecommons.org/licenses/by-nc-sa/4.0/). 